\title{
Cardiovascular risk in rheumatoid arthritis and diabetes: how does it compare and when does it start?
}

\author{
Michael T Nurmohamed, ${ }^{1-3}$ George Kitas ${ }^{1,3}$
}

\begin{abstract}
There is a substantial amount of evidence for an increased cardiovascular risk in rheumatoid arthritis (RA), 1.5-2fold in comparison with the general population, ${ }^{1}{ }^{2}$ but large-scale studies are still lacking. The majority of the evidence originates from several decades ago, a different era of natural history and management of RA, and thus there is still an unmet need for contemporary large epidemiological studies addressing the magnitude of the cardiovascular risk in RA. These studies should also address if and to what extent the cardiovascular risk in RA is comparable to that of diabetes, an established paradigm for a doubled cardiovascular risk, ${ }^{3}$ as recent studies have pointed towards this direction. ${ }^{4}$ If confirmed, then this may underpin the need for cardiovascular risk prevention and management in patients with RA, and a possible approach towards achieving this in everyday practice.
\end{abstract}

\section{CARDIOVASCULAR RISK IN RA VERSUS DIABETES: CARRÉ STUDY}

In a prospective Dutch study, the magnitude of the enhanced cardiovascular riskin RA was investigated by comparing prevalent cardiovascular disease (CVD) with that of diabetes. ${ }^{5}$ The study comprised 353 RA patients (diagnosed between 1989 and 2001, aged between 50 and 75 years; the CARdiovascular research in RhEumatoid arthritis (CARRÉ) study), and participants of a population-based cohort study on CVD and its risk factors (Hoorn study). Prevalent CVD was

\footnotetext{
'Department of Internal Medicine, VU University Medical Centre, Jan van Breemen Research Institute, Amsterdam, The Netherlands

2Department of Rheumatology, VU University

Medical Centre, Jan van Breemen Research Institute, Amsterdam, The Netherlands

${ }^{3}$ Department of Rheumatology, University of

Manchester, Russells Hall Hospital, Manchester, UK
}

Correspondence to Dr Michael T Nurmohamed, Departments of Internal Medicine and Rheumatology, VU University Medical Centre, PO Box 7057,

Amsterdam 1007MB, The Netherlands; mt.nurmohamed@vumc.nl observed in $13 \%$ patients with RA, $5 \%$ in individuals without diabetes and $12 \%$ in individuals with type 2 diabetes ( $\mathrm{n}=194)$, respectively. The OR for CVD, adjusted for age and sex, were 3.1 in RA patients and 2.3 in individuals with type 2 diabetes in comparison with individuals without diabetes. Adjustment for (other) cardiovascular risk factors only slightly attenuated the $\mathrm{OR}$, indicating that RA itself is an independent CVD risk factor. The prospective part of the investigation showed cardiovascular events in $9 \%$ of the RA patients and in $4 \%$ of the individuals without diabetes from the general population. ${ }^{6}$ The incidence rates were 3.1 per 100 patient-years for RA patients, and 1.5 per 100 person-years for individuals from the general population. The age and sex-adjusted relative risk was 2.0 with almost no change after adjusting for cardiovascular risk factors. Altogether, the CARRÉ study suggests that the cardiovascular risk in RA is increased to an extent that is comparable to that of diabetes, but confirmatory data from larger groups of patients are clearly needed.

\section{CARDIOVASCULAR RISK IN RA VERSUS DIABETES: NATIONWIDE DANISH STUDY}

In this issue of the journal, Lindhardsen et $\mathrm{al}^{7}$ conducted a nationwide study to investigate whether the risk of myocardial infarction (MI) in patients with RA is comparable to the risk in patients with diabetes (see page 929). The study comprised the whole Danish population older than 16 years and this cohort was followed during a 10-year period until 31 December 2006. By coupling nationwide registers, subjects with new-onset $R A$, new-onset diabetes and subjects developing a new (first) MI were identified. The cohort included more than 4300000 persons, of whom approximately 10500 developed RA and 130000 diabetes. The overall, fully adjusted, incidence rate ratio (IRR) of MI in RA was 1.7 (95\% CI 1.5 to 1.9 ), which was completely comparable to the risk in diabetes: IRR
1.7 (95\% CI 1.6 to 1.8$)$. The IRR was age dependent, particularly in women younger than 50 years, who had a 5.5fold increased risk. At the start of the study, 19377 patients with an RA diagnosis were excluded indicating a prevalence of approximately $0.045 \%$, and this appears low in comparison with other European countries, where it is approximately $1 \%$. This could be due to either underreporting or a true lower prevalence in Denmark. Moreover, RA diagnosis was defined according to International Classification of Diseases codes in combination with prescription of disease-modifying antirheumatic drugs. Therefore, the observed results most likely do not apply to mild cases of RA, treated with non-steroidal anti-inflammatory drugs only. Similarly, the prevalence of diabetes appears low in comparison with the incidence of diabetes $(1.8 \%$ and $3.0 \%$, respectively) lower than the overall diabetes prevalence of $4.2 \%$ in Denmark. ${ }^{8}$ However, as argued by the authors, at present an earlier diagnosis of diabetes and more intensive antidiabetic therapy are plausible explanations for this apparent discrepancy between prevalence and incidence. Nevertheless, these results are clearly relevant, as the RA patients encountered in our daily rheumatological practice resemble the RA population studied by Lindhardsen et al. ${ }^{7}$

When comparing the MI rates between the two studies then there is a striking difference: the incidence of MI was approximately $0.6 / 100$ patient-years in the Danish study versus approximately 2.3/100 patient-years in the CARRÉstudy. Moreover, another recently published study revealed a rate of approximately $0.2 / 100$ patient-years. ${ }^{9}$ Methodological issues might (partly) explain these striking differences, but also that MI rates differs across populations. Obviously, these findings will have power implications for cardiovascular intervention trials in RA.

\section{WHEN DOES THE INCREASED CARDIOVASCULAR RISK IN RA START?}

Carotid artery intima media thickness (IMT) is an important marker for early, preclinical, atherosclerosis and a predictor of future cardiovascular events in the general population, ${ }^{10}$ but this has not yet been shown in RA. However, several investigations have studied IMT in patients with RA, and a recent metaanalysis of 22 studies, encompassing 1384 RA patients and 1147 controls, revealed an overall greater carotid IMT in RA of $0.09 \mathrm{~mm}(95 \%$ CI 0.07 to $0.11 \mathrm{~mm}) .{ }^{11} \mathrm{As}$ 
disease characteristics, such as disease duration, were not related to carotid IMT this would imply that there was already an increased IMT early in the disease. However, the number of studies with early RA patients was small and comprised a limited number of patients. Moreover, a more recent study in 79 early RA patients did not demonstrate an increased IMT. ${ }^{12}$

Using general population norms, the observed carotid IMT difference of $0.09 \mathrm{~mm}$ of the meta-analysis of RA studies would indicate an approximately $15 \%$ increased cardiovascular risk, ${ }^{13}$ and this is significantly less than expected, compared with the approximately doubled cardiovascular risk in RA indicated by the hard endpoint epidemiological studies. As the majority of cardiovascular events are caused by plaque instability and rupture this may imply that, in a 'high-grade' inflammatory state, such as this of RA, plaques may rupture more easily and earlier than in a noninflammatory situation. ${ }^{14}$

It is important to note that dyslipidaemia is already present in the preclinical phase of RA. In a recent controlled study of 79 subjects who later developed RA, the lipid profile over time and its relationship with inflammation and serological markers was studied. ${ }^{15}$ The lipid profile displayed, on average, 4\% higher total cholesterol, 9\% lower high-density lipoprotein cholesterol and 17\% higher triglyceride levels compared with matched controls ( $p \leq 0.05)$, at least 10 years before the onset of RA symptoms, indicating an approximately $15-20 \%$ increased risk, which is remarkably similar to the increased risk extrapolated from the meta-analysis.

Altogether there appears to be an increased cardiovascular risk already at disease onset of RA, that is further substantiated by the findings of Lindhardsen et $\mathrm{al}^{7}$ and others. ${ }^{16}$ In contrast, several other reports indicate that a clear excess of cardiovascular risk is only observed after approximately 10 years of RA disease duration. ${ }^{17} 18$ One of the reasons for this discrepancy might be that at disease onset there is a limited increased risk that is further amplified by clinically overt RA, leading to an increased slope of cardiovascular events against time. This widening difference with the general population may differ between investigations, resulting in an earlier or later detection of the enhanced cardiovascular burden in comparison with the general population. This could explain why some epidemiological studies of incident (early) RA have not demonstrated a markedly increased cardiovascular risk, whereas the majority of studies of prevalent (established) RA have clearly shown such an excess. ${ }^{19}$

\section{CARDIOVASCULAR RISK MANAGEMENT IN RA: WHEN AND HOW?}

There is currently little doubt that the cardiovascular risk in RA is substantially elevated in comparison with the general population, and that it is comparable to patients with diabetes. There are several suggestions, including the study of Lindhardsen et al, ${ }^{7}$ that the RR is higher in younger age groups, but as the absolute risk is much higher in older age groups, preventive cardiovascular strategies should not be restricted to a particular age group.

There are several pivotal steps in cardiovascular risk management in RA. The first is acknowledging that this risk exists, and unfortunately it does not appear that this realisation has been consistently translated into routine clinical practice in many parts of the world. The second step involves the determination of the cardiovascular risk profile (including at least an assessment of blood pressure, smoking status and lipid profile) at regular intervals. On the basis of these and other easily accessible data (eg, age, sex, family history of premature coronary heart disease etc.) and the aid of calculators such as Framingham and SCORE, the 10-year cardiovascular risk of a particular person can be calculated. Primary prevention involving treatment with statins and/ or antihypertensive agents is only indicated in the general population when this 10 -year risk is above a certain value. For instance, in The Netherlands this would be a 10 -year cardiovascular mortality risk of $10 \%$ or more, whereas in the UK this would be a 10 -year cardiovascular morbidity risk of $20 \%$ or more. The third step is to make sure that such primary prevention therapy is successfully implemented, ie, that predetermined targets of blood pressure and cholesterol are achieved (although it is still uncertain what exactly these targets should be for RA patients, those accepted in the general population should be aimed for at the least) — there is much evidence that this is not happening, either in the management of blood pressure $^{20}$ or dyslipidaemia ${ }^{21}$ in patients with RA. Finally, tight RA disease control is likely to decrease cardiovascular risk, but the effects of antirheumatic medications on individual cardiovascular risk factors need to be regularly assessed. ${ }^{22}$ The task force of the European League Against
Rheumatism (EULAR) has recently issued 10 recommendations regarding cardiovascular risk management in patients with RA and other types of inflammatory arthritis, ${ }^{23}$ several aspects of which warrant comment. First, as the cardiovascular risk appears to be increased even before disease onset it is necessary to screen all patients with RA. Second, the existing risk models applied to the general population underestimate the true cardiovascular risk in RA. Therefore, the EULAR task force advocated the use of a 1.5 multiplication factor when two out of three of the following characteristics are present: disease duration of 10 years or more, the presence of extra-articular manifestations or the presence of rheumatoid factor or anti-cyclic citrullinated peptide antibodies. This recommendation was based on the existing literature until May 2008, and since then there have been some reports advocating alternative approaches, for example, to increase the age of an RA patient by 10 years to get a more precise cardiovascular risk estimate, to use other risk scores, such as the Reynold's risk score, which incorporates C-reactive protein in the risk algorithm or the QRISK2 algorithm, which includes RA (like diabetes) as an independent risk factor. ${ }^{24} 25$ Obviously, any of these approaches (including the EULAR recommendations) should be fully validated and the discussion will only be solved by the development of an RA-specific cardiovascular risk prediction model through specifically designed prospective studies, which will take several years to complete. The EULAR task force clearly underscored the need for further research in this field, including the development of RA-specific cardiovascular risk algorithms as well as the need for hard cardiovascular endpoint trials with statins and/ or antihypertensive agents in RA.

\section{CONCLUSIONS}

If there was any doubt or debate about an enhanced cardiovascular risk in RA, the findings of Lindhardsen et al ${ }^{7}$ clearly settles this. Importantly, they also provide further evidence that the cardiovascular risk in RA is broadly similar to that of contemporarily managed diabetes. In this respect is important to note that the cardiovascular risk in diabetes has decreased during recent decades probably as a result of cardiovascular risk management with statins and antihypertensive agents. ${ }^{26}$

The results of the study by Lindhardsen et $\mathrm{al}^{7}$ as well as the success of cardiovascular risk management in diabetes provides a clear incentive to identify and actively 
manage, if necessary, cardiovascular risk in all RA patients as part of quality routine rheumatological practice. This is particularly important, as, for instance, the need for cardiovascular risk management is hardly acknowledged in primary care. ${ }^{27}$ The EULAR recommendations provide a useful framework towards achieving cardiovascular risk management, but they also clearly acknowledge the need for further research, including hard cardiovascular endpoint primary prevention trials specifically in patients with RA.

\section{Competing interests None.}

Contributors MTN and GK both contributed to writing this editorial.

Provenance and peer review Commissioned; externally peer reviewed.

Accecpted 13 March 2011

Published Online First 30 March 2011

Ann Rheum Dis 2011;70:881-883

doi:10.1136/ard.2010.145839

\section{REFERENCES}

1. Van Doornum S, McColl G, Wicks IP. Accelerated atherosclerosis: an extraarticular feature of rheumatoid arthritis? Arthritis Rheum 2002:46:862-73.

2. Bacon PA, Stevens RJ, Carruthers DM, et al. Accelerated atherogenesis in autoimmune rheumatic diseases. Autoimmun Rev 2002;1:338-47.

3. Sarwar N, Gao P, Seshasai SR, et al. Diabetes mellitus, fasting blood glucose concentration, and risk of vascular disease: a collaborative meta-analysis of 102 prospective studies. Lancet 2010;375:2215-22.

4. Peters MJ, van Halm VP, Voskuyl AE, et al. Does rheumatoid arthritis equal diabetes mellitus as an independent risk factor for cardiovascular disease? A prospective study. Arthritis Rheum 2009;61:1571-9.

5. Stamatelopoulos KS, Kitas GD, Papamichael CM, et al. Atherosclerosis in rheumatoid arthritis versus diabetes: a comparative study. Arterioscler Thromb Vasc Biol 2009;29:1702-8.
6. van Halm VP, Peters MJ, Voskuyl AE, et al. Rheumatoid arthritis versus diabetes as a risk factor for cardiovascular disease: a cross-sectional study, the CARRE Investigation. Ann Rheum Dis 2009;68:1395-400.

7. Lindhardsen J, Ahlehoff 0 , Gislason GH, et al. The risk of myocardial infarction in rheumatoid arthritis and diabetes mellitus: a Danish nationwide cohort study. Ann Rheum Dis 2011;70:929-34.

8. Carstensen B, Kristensen JK, Ottosen P, et al.; Steering Group of the National Diabetes Register. The Danish National Diabetes Register: trends in incidence, prevalence and mortality. Diabetologia 2008:51:2187-96.

9. Greenberg JD, Kremer JM, Curtis JR, et al.; on behalf of the CORRONA Investigators. Tumour necrosis factor antagonist use and associated risk reduction of cardiovascular events among patients with rheumatoid arthritis. Ann Rheum Dis 2011;70:576-82.

10. O'Leary DH, Polak JF, Kronmal RA, et al. Carotidartery intima and media thickness as a risk factor for myocardial infarction and stroke in older adults. Cardiovascular Health Study Collaborative Research Group. N Engl J Med 1999;340:14-22

11. van Sijl AM, Peters MJ, Knol DK, et al. Carotid intima media thickness in rheumatoid arthritis as compared to control subjects: a meta-analysis. Semin Arthritis Rheum. Published Online First: 1 October 2010. PMID: 20889191.

12. Södergren A, Karp K, Boman K, et al. Atherosclerosis in early rheumatoid arthritis: very early endothelial activation and rapid progression of intima media thickness. Arthritis Res Ther 2010;12:R158

13. Bots ML, Hoes AW, Koudstaal PJ, et al. Common carotid intima-media thickness and risk of stroke and myocardial infarction: the Rotterdam Study. Circulation 1997;96:1432-7.

14. Aubry MC, Maradit-Kremers H, Reinalda MS, et al. Differences in atherosclerotic coronary heart disease between subjects with and without rheumatoid arthritis. J Rheumato 2007;34:937-42.

15. van Halm VP, Nielen MM, Nurmohamed MT, et al. Lipids and inflammation: serial measurements of the lipid profile of blood donors who later developed rheumatoid arthritis. Ann Rheum Dis 2007; 66:184-8

16. Holmqvist ME, Wedrén S, Jacobsson LT, et al. Rapid increase in myocardial infarction risk following diagnosis of rheumatoid arthritis amongst patients diagnosed between 1995 and 2006 J Intern Med 2010:268:578-85.

17. Kroot EJ, van Gestel AM, Swinkels HL, et al. Chronic comorbidity in patients with early rheumatoid arthritis: a descriptive study. J Rheumatol 2001;28:1511-17.

18. Aviña-Zubieta JA, Choi HK, Sadatsafavi M, et al. Risk of cardiovascular mortality in patients with rheumatoid arthritis: a meta-analysis of observational studies. Arthritis Rheum 2008:59:1690-7.

19. Kitas GD, Erb N. Tackling ischaemic heart disease in rheumatoid arthritis. Rheumatology (Oxford) 2003;42:607-13.

20. Panoulas VF, Douglas KM, Milionis HJ, et al. Prevalence and associations of hypertension and its control in patients with rheumatoid arthritis. Rheumatology (Oxford) 2007;46:1477-82.

21. Toms TE, Panoulas VF, Douglas KM, et al. Statin use in rheumatoid arthritis in relation to actual cardiovascular risk: evidence for substantial undertreatment of lipid-associated cardiovascular risk? Ann Rheum Dis 2010;69:683-8.

22. Kitas GD, Gabriel SE. Cardiovascular disease in rheumatoid arthritis: state of the art and future perspectives. Ann Rheum Dis 2011;70:8-14.

23. Peters MJ, Symmons DP, McCarey D, et al. EULAR evidence-based recommendations for cardiovascular risk management in patients with rheumatoid arthritis and other forms of inflammatory arthritis. Ann Rheum Dis 2010;69:325-31.

24. Ridker PM, Paynter NP, Rifai N, et al. C-reactive protein and parental history improve global cardiovascular risk prediction: the Reynolds Risk Score for men. Circulation 2008;118:2243-51.

25. Hippisley-Cox J, Coupland C, Vinogradova Y, et al. Predicting cardiovascular risk in England and Wales: prospective derivation and validation of QRISK2. BMJ 2008:336:1475-82.

26. Seshasai SR, Kaptoge S, Thompson A, et al. Diabetes mellitus, fasting glucose, and risk of cause-specific death. N Engl J Med 2011;364:829-41.

27. Bell C, Rowe IF. The recognition and assessment of cardiovascular risk in people with rheumatoid arthritis in primary care: a questionnaire-based study of general practitioners. Musculoskeletal Care. Published Online First: 24 November 2010. PMID: 21110305. 